\title{
Troponins in cardiac amyloidosis: multipurpose markers
}

\author{
Federico Perfetto, Franco Bergesio, Michele Emdin and Francesco Cappelli
}

We read the Review by Giannitsis and Katus (Giannitsis, E. \& Katus, H. Cardiac troponin level elevations not related to acute coronary syndromes. Nat. Rev. Cardiol. 10, 623-634 [2013]) ${ }^{1}$ with great interest. We believe that a few points need to be discussed in greater detail. We were surprised to note the absence of any mention of amyloidosis among the chronic conditions not related to acute coronary syndrome (ACS) that can be associated with substantial elevation in cardiac troponin (cTn) levels.

Light chain amyloidosis is a plasma cell dyscrasia characterized by extracellular deposition of toxic $\beta$-fibrillar immunoglobulin light chain in several organs, including the heart. ${ }^{2}$ Cardiac biomarkers, including cTn, have been studied extensively in light chain amyloidosis, because cardiac involvement is the most-important determinant of clinical outcome. ${ }^{3}$ Persistently elevated cTn levels are frequently found in patients with amyloidosis, and are an indication of cardiac amyloid infiltration. ${ }^{2}$ In our experience, cTnI levels $>0.09 \mu \mathrm{g} / \mathrm{l}$ at presentation were recorded in $34 \%$ of 64 patients with light chain amyloidosis, and in $58 \%$ of 38 patients with transthyretin-related cardiac amyloidosis (F. Perfetto, unpublished work). Notably, patients with amyloidosis often present with misleading symptoms and signs, such as chest pain or a psuedoinfarction pattern (QS waves in consecutive leads) on the electrocardiogram, which can suggest a diagnosis of ischaemic heart disease, as well as regional radionuclide uptake defects on myocardial perfusion scintigraphy, owing to severe, localized amyloid infiltration. Patients with cardiac amyloidosis, therefore, often undergo unnecessary coronary angiography, which usually shows normal epicardial arteries. ${ }^{3-5}$ Interestingly, serial cTn assays in patients with amyloidosis do not show a crescendo-decrescendo pattern of elevation. ${ }^{6}$ This feature distinguishes amyloidosis from other causes of cTn elevation.
cTn level is also a prognostic factor in cardiac light chain amyloidosis. A cTnT level $>0.035 \mathrm{~g} / \mathrm{l}$ accurately predicts poor survival, ${ }^{7}$ and patients with cTnT $>0.060 \mathrm{~g} / 1$ or $\mathrm{c} \operatorname{TnI}>0.10 \mu \mathrm{g} / \mathrm{l}$ have an increased risk of mortality associated with blood stem cell transplantation, despite adjustment of melphalan dose. ${ }^{7,8}$ In a cohort of 76 consecutive patients with cardiac light chain amyloidosis, we found that $\mathrm{cTnI} \geq 0.085 \mu \mathrm{g} / \mathrm{l}$ predicted right ventricular amyloid involvement, an independent negative prognostic factor. ${ }^{9}$ Interestingly, these data show that cTnI has greater specificity and sensitivity than $\mathrm{N}$-terminal proB-type natriuretic peptide for predicting right ventricular dysfunction. ${ }^{9}$

The exact pathological mechanism of cTn release in patients with amyloidosis is uncertain, but is likely to be multifactorial, including microvascular ischaemia, the mechanical effects of amyloid infiltration, and the proinflammatory or toxic effects of the amyloid protein or its precursor, leading to myocardial cell damage with membrane leakage and troponin release. ${ }^{10}$ The presence of late gadolinium enhancement on cardiac MRI in patients with amyloidosis has been associated with increased cTn levels, suggesting a correlation between biomarker elevation and advanced amyloid infiltration. ${ }^{11}$ For these reasons, we believe that amyloidosis should be included among the conditions not related to ACS that are associated with chronically elevated cTn levels.

Future perspectives for the use of cTn assays in the setting of amyloidosis include screening for silent cardiac involvement in patients with conditions that confer a high risk of developing cardiac amyloidosis, such as monoclonal gammopathies of undetermined significance, or carriers of amyloidogenic mutation; for determining optimal treatment; and for evaluating responses to therapy. Studies addressing these specific applications of cTn measurement are needed.
Regional Amyloid Centre, Azienda OspedalieroUniversitaria Careggi, Largo Giovanni Alessandro Brambilla 3, 50134 Florence, Italy (F. Perfetto, F. Bergesio, F. Cappelli). Fondazione Toscana Gabriele Monasterio, Via Giuseppe Moruzzi 1, 56124 Pisa, Italy (M. Emdin). Correspondence to: F. Perfetto federico.perfetto@unifi.it

\section{Competing interests}

The authors declare no competing interests.

1. Giannitsis, E. \& Katus, H. Cardiac troponin level elevations not related to acute coronary syndromes. Nat. Rev. Cardiol. 10, 623-634 (2013).

2. Perfetto, F. et al. Cardiac amyloidosis: the heart of the matter. Intern. Emerg. Med. 8, 191-203 (2013).

3. Palladini, G. et al. The combination of highsensitivity cardiac troponin T (hs-cTnT) at presentation and changes in $\mathrm{N}$-terminal natriuretic peptide type B (NT-proBNP) after chemotherapy best predicts survival in $\mathrm{AL}$ amyloidosis. Blood 116, 3426-3430 (2010).

4. Mueller, P. S., Edwards, W. D. \& Gertz, M. A Symptomatic ischemic heart disease resulting from obstructive intramural coronary amyloidosis. Am. J. Med. 109, 181-188 (2000).

5. Miani, D., Rocco, M., Alberti, E., Spedicato, L. \& Fioretti, P. M. Amyloidosis of epicardial and intramural coronary arteries as an unusual cause of myocardial infarction and refractory angina pectoris. Ital. Heart J. 3, 479-482 (2002).

6. Zabernigg, A., Schranzhofer, R., Kreczy, A. \& Gattringer, K. Continuously elevated cardiac troponin I in two patients with multiple myeloma and fatal cardiac amyloidosis. Ann. Oncol. 14, 1791 (2003).

7. Dispenzieri, A. et al. Prognostication of survival using cardiac troponins and N-terminal probrain natriuretic peptide in patients with primary systemic amyloidosis undergoing peripheral blood stem cell transplantation. Blood 104, 1881-1887 (2004).

8. Gertz, M. et al. Troponin T level as an exclusion criterion for stem cell transplantation in lightchain amyloidosis. Leuk. Lymphoma 49, 36-41 (2008).

9. Cappelli, F. et al. Biohumoral markers as predictor of right ventricular dysfunction in $\mathrm{AL}$ amyloidosis. Amyloid (in press).

10. Capone, R., Amsterdam, E. A., Mason, D. T. $\&$ Zelis, R. Systemic amyloidosis, functional coronary insufficiency, and autonomic impairment. Ann. Intern. Med. 76, 599-603 (1972).

11. Syed, I. S. et al. Role of cardiac magnetic resonance imaging in the detection of cardiac amyloidosis. JACC Cardiovasc. Imaging $\mathbf{3}$, 155-164 (2010). 